\title{
Design of Vibration Signal Acquisition System for Hydraulic Steel Gate
}

\author{
ZhongYuan Jing, ChuanWu Zhang \\ School of electrical information engineering, Southwest Minzu University, Chengdu, Sichuan 610041, China
}

Email: 1522452618@qq.com

\begin{abstract}
Aiming at the demand of real-time monitoring of gate vibration problems of hydropower stations, the steel gate vibration monitoring system was designed and implemented. The system collects the vibration signal through the acceleration sensor, and adjusts and amplifies the weak signal. The STM32F103ZET6 is used as the control processing core, which controls the A/D conversion and transmits the data to the memory through DMA, and then transmits the data to the computer through the serial port communication. The design of the ADXL356 accelerometer and the design of the signal conditioning amplifier circuit and the software design of the acquisition system are highlighted. The analysis of the experimental results shows that the system can achieve high-precision acquisition of signals, which can provide technical reference for the online monitoring system of gates.
\end{abstract}

Keywords: STM32F103ZET6, acceleration transducer, A/D conversion, serial communications

\section{Introduction}

With the development of the economy, the construction of the Gaoba Reservoir has been on the rise in recent years. As a basic component of hydraulic structures, the operation of steel gates under various working conditions directly affects the overall safety of the dam. In the water conservancy and hydropower industry, hydraulic metal equipment will produce different degrees of vibration due to the external force. This uninterrupted vibration is likely to cause fatigue of the components, which is not conducive to the long-term safe operation of the equipment. When a steel gate operates under dynamic water load, the submerged water jump behind the gate periodically impacts the gate, and inevitably meets the "water hammer effect" of dynamic pressure. This coupling phenomenon may cause the change of vibration characteristics of the gate structure, which has adverse effects on its dynamic stability[1-3]. In this paper, a real-time online monitoring system is designed for the vibration acceleration of the gate under various working conditions, in order to provide reference for the safe operation of the gate.

\section{Overall Design of the System}

This system includes hardware and software design. The design of the peripheral circuit of the acceleration sensor, driving circuits, signal conditioning circuit and amplifying circuit are given in hardware design part and flow chart of the software and algorithm are also given in software design part.

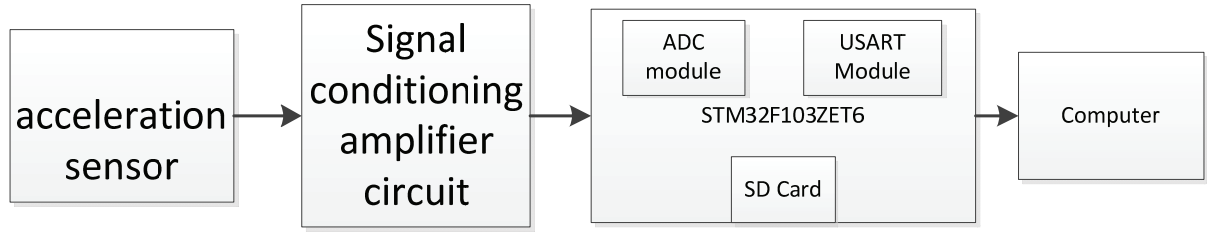

Figure1. Overall design of the acquisition system

The system collects the vibration signal from the acceleration sensor, and then the signal is processed by the signal conditioning circuit to filter out part of the noise signal and the mixed DC component and amplify the signal. The amplification circuit includes a constant voltage constant current circuit and a 
differential amplification circuit. After that, the signal is converted by AD under the control of STM32F103ZET6 and transmitted to the computer through serial communication. The overall design block diagram of the system is shown in Figure 1.

\section{Hardware Design of the System}

\subsection{Introduction of ADXL356 and Design of Acceleration Sensor Circuit}

The ADXL356 MEMS accelerometer is the latest example of ADI's high-performance sensor technology, providing high-quality data for IoT (Internet of Things) applications and enabling intelligent detection from the edge of the network. The analog output ADXL356 is low noise density, low $0 \mathrm{~g}$ offset drift, low power, 3 -axis accelerometers with selectable measurement ranges ${ }^{[4]}$.

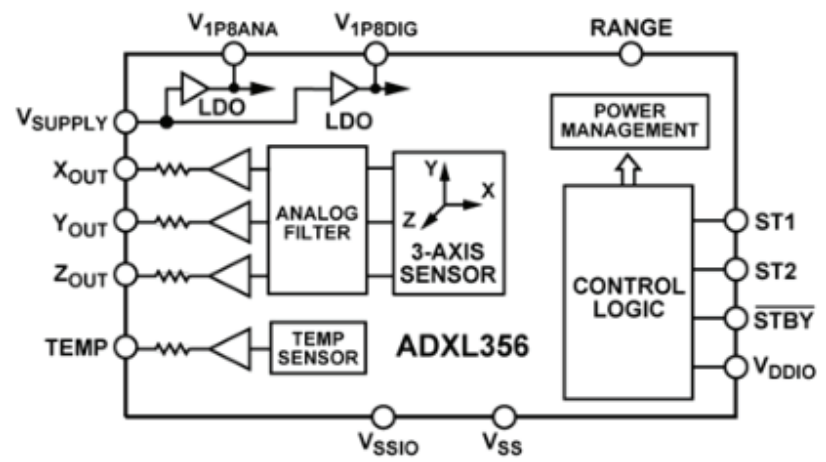

Figure2. ADXL356

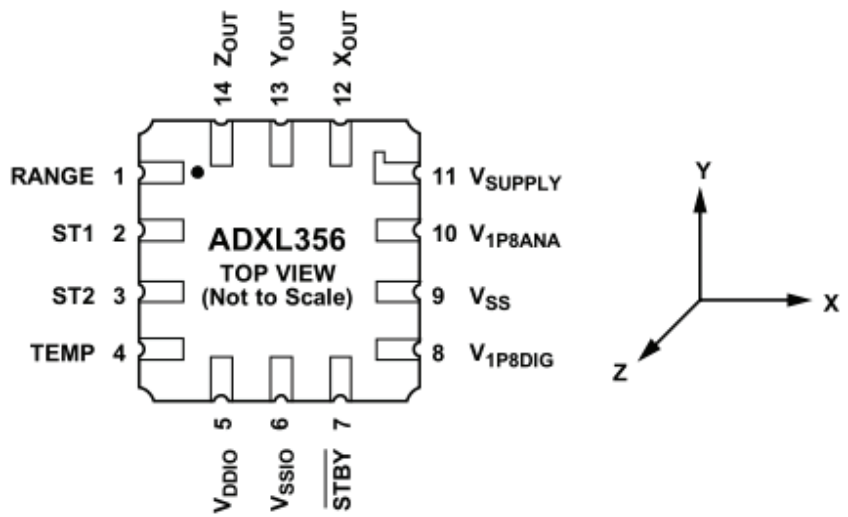

Figure3. ADXL356 pin configuration

\section{FEATURES}

- Hermetic package offers excellent long-term stability

- 0 g offset vs. temperature (all axes): $0.75 \mathrm{mg} / \mathrm{C}$ maximum

- Ultralow noise density (all axes): $80 \mu \mathrm{g} / \sqrt{ } \mathrm{Hz}$

- Low power, Vsupply (LDO enabled)

- Measurement mode: $150 \mu \mathrm{A}$

- Standby mode: $21 \mu \mathrm{A}$

- User adjustable analog output bandwidth

- Data interpolation routine for synchronous sampling

- Integrated temperature sensor 
- Integrated temperature sensor

- Voltage range options

○ V Supply with internal regulators: $2.25 \mathrm{~V}$ to $3.6 \mathrm{~V}$

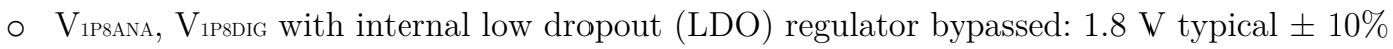

- 14-terminal, $6.0 \mathrm{~mm} \times 5.6 \mathrm{~mm} \times 2.05 \mathrm{~mm}$, LCC package, 0.26 grams

The ADXL356 offer industry leading noise, minimal offset drift over temperature, and long-term stability, enabling precision applications with minimal calibration. Sensor sensitivity is an important index of static characteristics of sensors. For a linear sensor such as an accelerometer, its sensitivity is the slope of its static characteristic, which is theoretically constant.

The power supply voltage of ADXL356 is between $2.25 \mathrm{~V}$ and $3.6 \mathrm{~V}$. When the power supply voltage changes in this range, it directly affects the sensitivity of the sensor. The parameters of the acceleration sensor designed in this system are measured and nominated under the condition of $3.3 \mathrm{~V}$ power supply voltage. The sensitivities of the output signals of X-axis, Y-axis and Z-axis are $499 \mathrm{mV} / \mathrm{g}, 492 \mathrm{mV} / \mathrm{g}$ and $494 \mathrm{mV} / \mathrm{g}$ respectively after conditioning and amplification.

At the same time, the low $0 \mathrm{~g}$ bias output (the output voltage when the accelerattion is zero) is also an important reference parameter for the acceleration sensor. The $0 \mathrm{~g}$ bias output of ADXL356 is theoretically equal to V1P8ANA /2. For example, when the power supply voltage is 3.3V, the output voltage of $\mathrm{X}$-axis is $1.399 \mathrm{~V}$. According to $\mathrm{V} 1 \mathrm{P} 8 \mathrm{ANA}=1.8 \mathrm{~V}$, the peak-to-peak value of the $\mathrm{X}$-axis output is $499 \mathrm{mV} / \mathrm{g}$ after conditioning and amplification. It can be seen that the measured acceleration is $1 \mathrm{~g}$.

The ADXL356B has 14 pins, including self-test, common ground, power supply, and 12, 13, and 14 pins for acceleration analog signal output. In order to make the sensor work properly, some necessary auxiliary circuit elements need to be added to the periphery of the chip. Fig. 4 is a complete circuit diagram design.

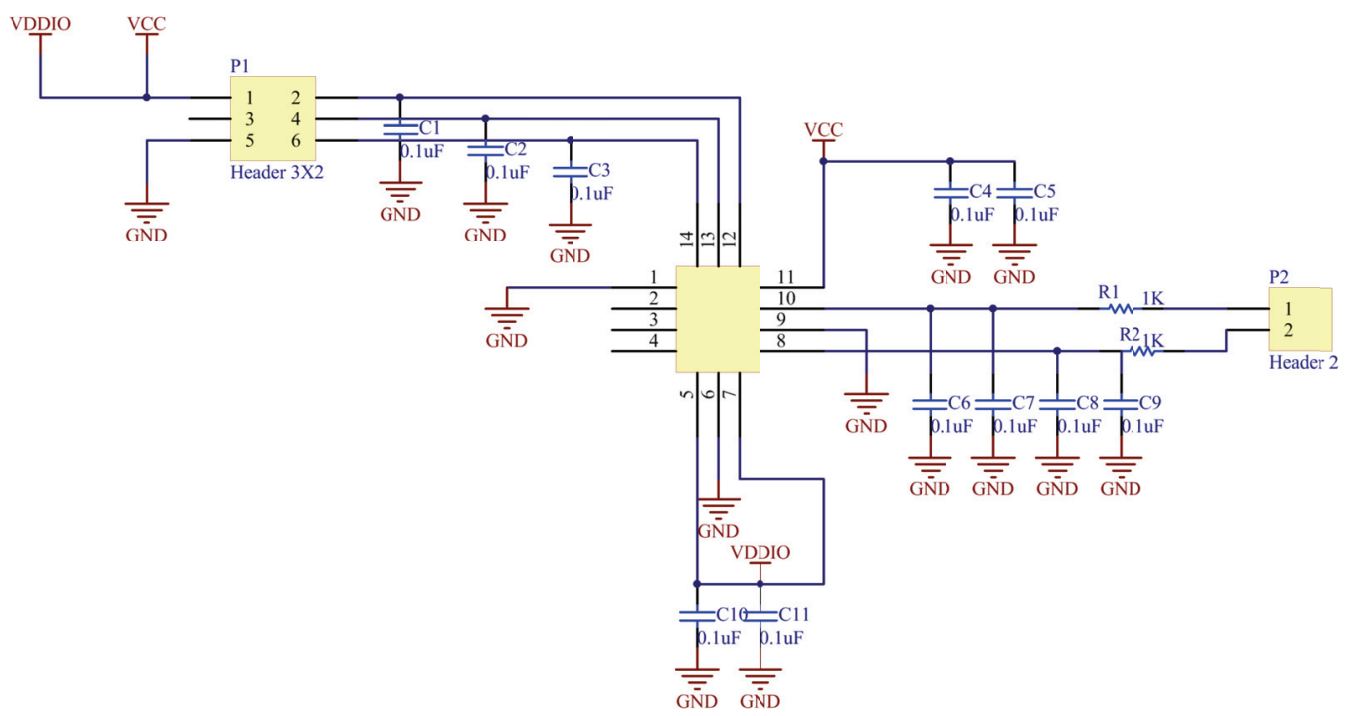

Figure 4. Circuit diagram of the acceleration sensor

\subsection{Design of Conditioning Circuit}

The original signal collected by the sensor is generally analog signal, which is not easy to process. The conditioning circuit is to filter, amplify, buffer or calibrate the weak electrical signal output by the sensor to make it suitable for the input of analog-to-digital converter. The chips involved in the design of the conditioning circuit of the system are LNA827, ADM8829, LP5907 and so on.

The ADM8829 is a charge-pump voltage inverter, which may be used to generate a negative supply from a positive input. Input voltages ranging from $+1.5 \mathrm{~V}$ to $+5.5 \mathrm{~V}$ can be inverted into a negative -1.5 $\mathrm{V}$ to $-5.5 \mathrm{~V}$ output supply. This inverting scheme is ideal for generating a negative rail in single power-supply systems. Only two small external capacitors are needed for the charge pump. Output currents up to $25 \mathrm{~mA}$ with greater than $99 \%$ efficiency are achievable. 
The INA827 is a low-power, wide-supply voltage instrumentation amplifier that can operate in both single and dual-supply configurations. A single external resistor sets the gain from 5 to 1000 . The device operates with a supply voltage between $2.7 \mathrm{~V}$ and $36 \mathrm{~V}$, and is available in an MSOP-8 package.

Designed to meet the needs of sensitive RF and analog circuits, the LP5907 provides low noise, high PSRR, low quiescent current, as well as low line and load transient response figures. Using new innovative design techniques, the LP5907 offers class leading noise performance without the need for a separate noise filter capacitor. The LP5907 is designed to perform with a single $1-\mu \mathrm{F}$ input capacitor and a single $1-\mu \mathrm{F}$ ceramic output capacitor. With a reasonable PCB layout, the single $1-\mu \mathrm{F}$ ceramic output capacitor can be placed up to $10 \mathrm{~cm}$ away from the LP5907 device.

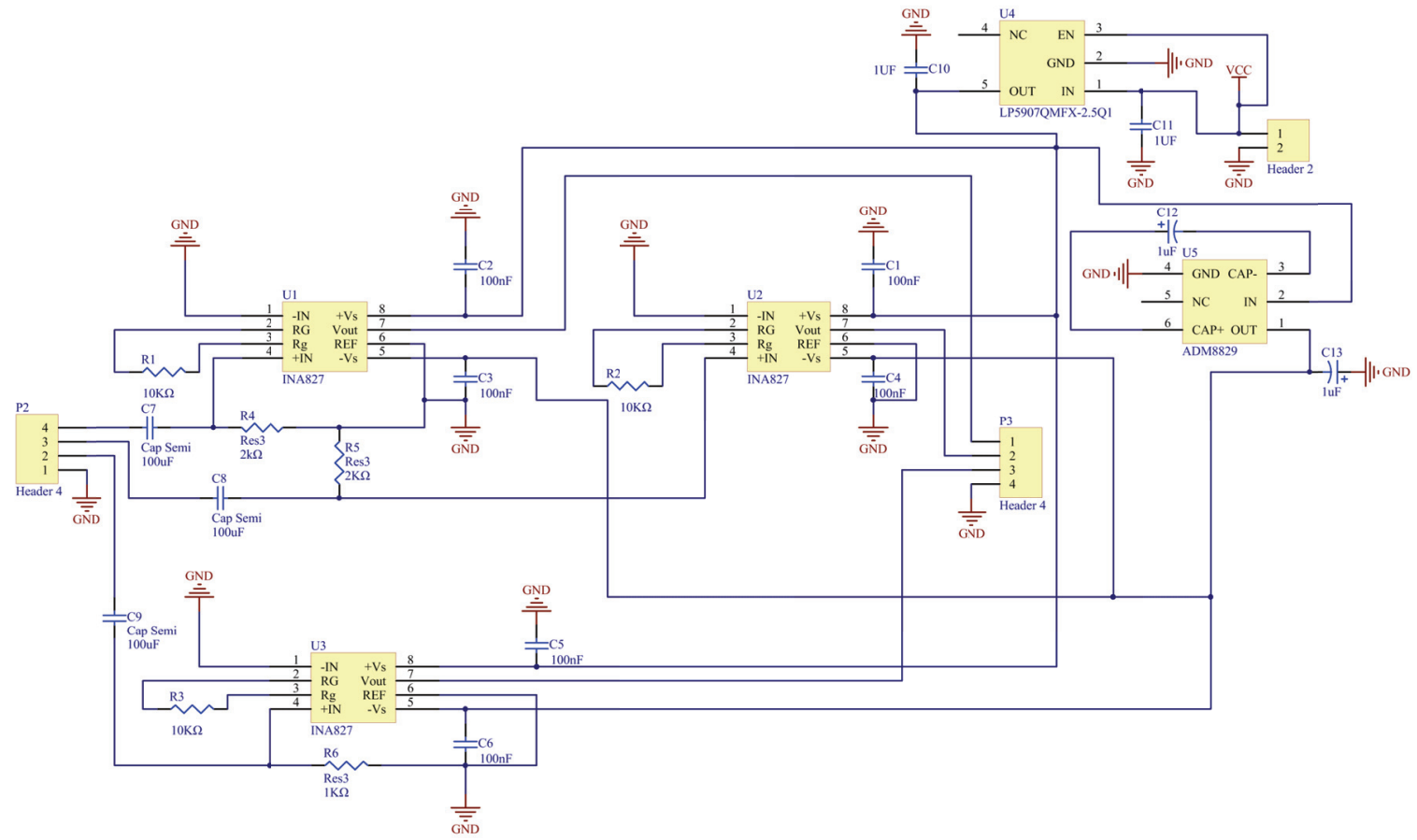

Figure 5. Schematic diagram of conditioning circuit

\subsection{Introduction of ADC in STM32F103ZET6}

The 32-bit Cortex-M3 microcontroller core based on ARMv7 architecture was introduced by ARM company, and then ST (Italian Semiconductor) company introduced MCU-STM32 microcontroller based on Cortex-M3 core. It has various communication interfaces, such as USART, I2C, SPI, etc. It can connect many sensors and control many devices ${ }^{[5]}$. The STM32F103ZET6 comes with 3 ADCs with 12-bit precision and up to 16 external channels per ADC. The ADC has many modes and is very powerful.

\section{Software Design of the System}

The Programming is completed in the development tool Keil MDK, mainly including the initialization of DMA and ADC, analog acquisition and transmission, and serial communication. The MCU needs to collect data in three directions of X, Y, and Z axes, so it requires three channels to collect simultaneously. The ADC acquisition channel should be set to scan continuous conversion mode because it is multi-channel simultaneous acquisition. The ADC acquisition data in the software program is transmitted by DMA. It is not only efficient but also convenient compared with reading datia in interrupt service function. The key point is to enable the DMA request of the ADC after initialization is completed. Using DMA to carry multi-channel data does not occupy CPU memory, so it is possible to process data while reading. Figure 6 is the program flow chart of the system software. The following part of the code: 

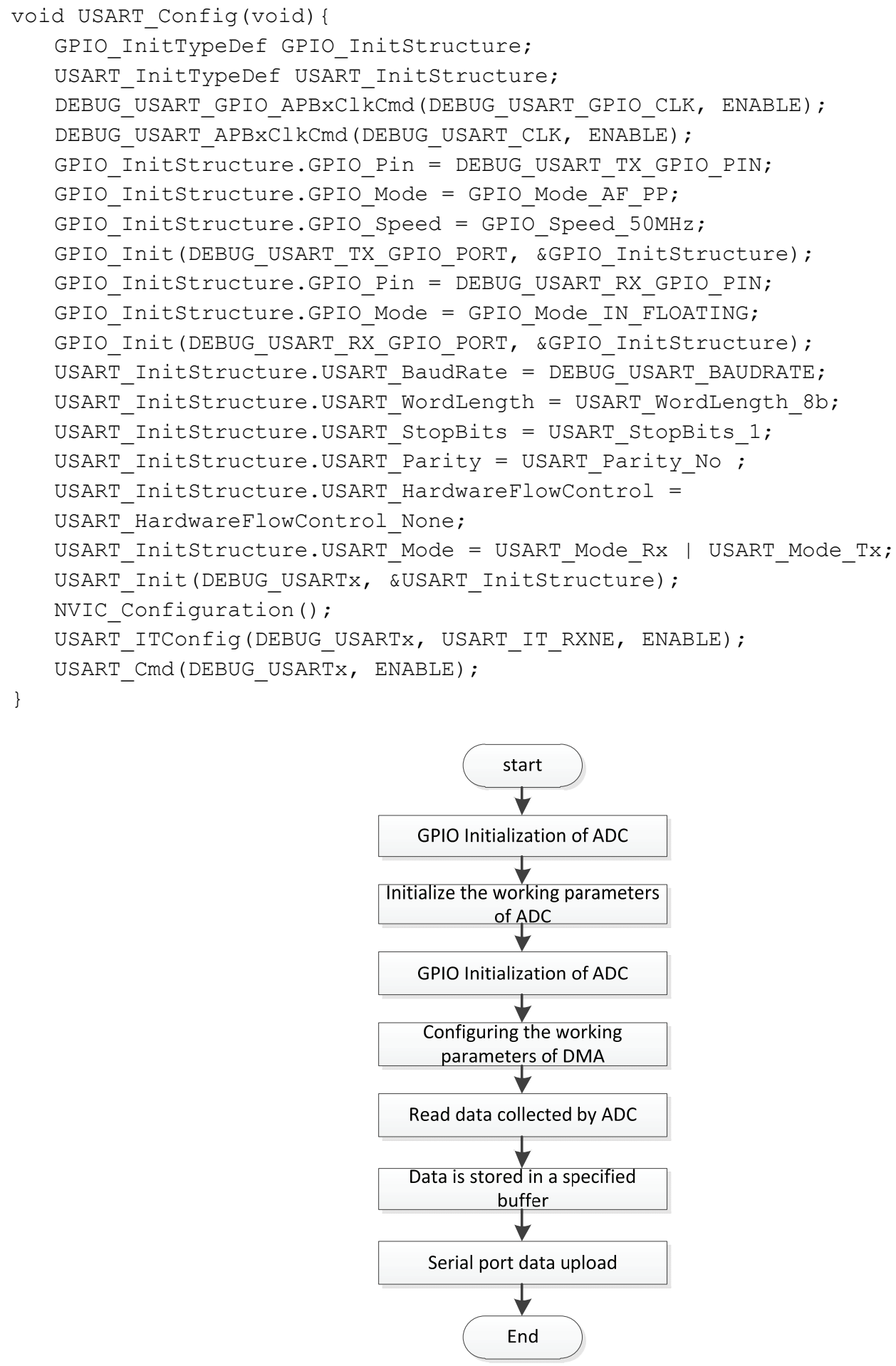

Figure 6. Main program flow chart

\section{$5 \quad$ Experiment Results and Analyses}

The experiment was carried out directly on the vibration calibration table DH1306. In order to evaluate the overall performance of the system more comprehensively, repeated experiments were carried out at different frequencies at the same acceleration and different accelerations at the same frequency. In the 
predetermined range $(\mathrm{g})$ and frequency range $(\mathrm{Hz})$, the Z-axis frequency response and amplitude linearity test results are shown in Table 1 and Table 2. It fully demonstrates that the system can achieve real-time on-line monitoring of vibration acceleration of steel gate.

Table 1. Acceleration of $1.0 \mathrm{~g}$ at different frequencies

\begin{tabular}{cccc}
\hline frequency $(\mathrm{Hz})$ & acceleration $(\mathrm{g})$ & output voltage $(\mathrm{V})$ & error $(\mathrm{dB})$ \\
\hline 20 & 1.0 & 1.400237 & 0.02 \\
30 & 1.0 & 1.397393 & -0.03 \\
40 & 1.0 & 1.399257 & 0.00 \\
50 & 1.0 & 1.398864 & -0.01 \\
60 & 1.0 & 1.398178 & -0.02 \\
70 & 1.0 & 1.397884 & -0.02 \\
\hline
\end{tabular}

Table 2. Frequency $40 \mathrm{~Hz}$ at different acceleration

\begin{tabular}{cccc}
\hline frequency $(\mathrm{Hz})$ & acceleration $(\mathrm{g})$ & output voltage $(\mathrm{V})$ & error $(\%)$ \\
\hline & 0.6 & 1.198081 & 0.21 \\
& 0.8 & 1.297511 & 0.23 \\
40 & 1.0 & 1.397181 & 0.23 \\
& 2.0 & 1.893824 & 0.32 \\
& 3.0 & 2.390114 & 0.45 \\
\hline
\end{tabular}

\section{Conclusion}

After many tests, it is proved that the vibration signal acquisition system of steel gate based on STM32 designed in this paper can meet the requirements of use.

The low-frequency accelerometer designed in this paper considers several factors that may affect the experimental results. The amplification circuit can adjust the amplification factor within a certain range, but the adjustment of the amplification factor involves a certain impact on the sensitivity and range of the sensor. The magnification of the design for the needs of the system is about 7 times. At present, the output value of the system is a voltage value, and the acceleration of the measured object can be calculated according to the voltage value. Subsequently, the program can be modified so that its output value is directly acceleration.

The hardware of the system designed in this paper is low complexity. Software development is relatively easy and widely applicable. The application value of this system is significant.

Acknowledgements. The work of this paper is supported by the Special Fund for Fundamental Research Operational Fees in Central Universities of Southwest Minzu University (Project number: CX2019SZ14).

\section{References}

1. Han Zhang, SU HuaiZhi, Chen Jian, et al. "Numerical analysis of dynamic characteristics of hydraulic radial cavity gate" Hydro-Science and Engineering, 2018(2):26-34.

2. Ma Bin,Guo Yiliang. "Current research status and development trend of hydraulic gate vibration". Hydro-Science and Engineering, 2019(2):55-64.

3. ZHANG WeiHua, YAN GenHua, CHENG FaZhan. Static and Dynamic Characteristics and Flow-induced Vibration of Deep-hole Arc Gate[J]. Hydro-Science and Engineering, 2016(2):111-119.

4. MENG WeiGuo. "Characteristics and Application of Tri-Axis Accelerometer ADXL330". International Electronic Elements, 2007(2):47-50.

5. YANG HuoLiang. "Practical Guidelines for STM32 Library Development (Second Edition)". 2017. 